University of Nebraska - Lincoln

DigitalCommons@University of Nebraska - Lincoln

$11-2004$

\title{
Screw Dislocation Interacting with Interfacial Edge-Cracks in Piezoelectric Bimaterial Strips
}

\author{
Xiangfa Wu \\ Department of Engineering Mechanics, University of Nebraska-Lincoln, xfwu@unlserve.unl.edu
}

Yuris A. Dzenis

Department of Engineering Mechanics, University of Nebraska-Lincoln, ydzenis@unl.edu

Bradley D. Rinschen

Department of Mechanical Engineering, University of Nebraska-Lincoln

Follow this and additional works at: https://digitalcommons.unl.edu/engineeringmechanicsfacpub

Part of the Mechanical Engineering Commons

Wu, Xiangfa; Dzenis, Yuris A.; and Rinschen, Bradley D., "Screw Dislocation Interacting with Interfacial Edge-Cracks in Piezoelectric Bimaterial Strips" (2004). Faculty Publications from the Department of Engineering Mechanics. 27.

https://digitalcommons.unl.edu/engineeringmechanicsfacpub/27

This Article is brought to you for free and open access by the Mechanical \& Materials Engineering, Department of at DigitalCommons@University of Nebraska - Lincoln. It has been accepted for inclusion in Faculty Publications from the Department of Engineering Mechanics by an authorized administrator of DigitalCommons@University of Nebraska - Lincoln. 


\title{
Screw Dislocation Interacting with Interfacial Edge-Cracks in Piezoelectric Bimaterial Strips
}

\author{
Xiang-Fa Wu, Yuris A. Dzenis \\ Department of Engineering Mechanics, Center for Materials Research and Analysis \\ University of Nebraska-Lincoln, Lincoln, NE 68588-0526, USA \\ Email:xfwu@unlserve.unl.edu (X.-F.Wu)
}

Bradley D. Rinschen

Department of Mechanical Engineering, University of Nebraska-Lincoln, Lincoln, NE 685880656, USA

\begin{abstract}
This paper is concerned with the interaction between an interfacial edge-crack and a screw dislocation under out-of-plane mechanical and in-plane electric loading in a piezoelectric bimaterial strip. In addition to a discontinuous electric potential across the slip plane, the dislocation is subjected to a line-force and a line-charge at the core. Under the framework of linear piezoelectricity, the out-of-plane displacement and in-plane electric potentials are constructed in closed-form by means of conformal mapping technique and the known solution for screw dislocation in cracked piezoelectric bimaterial. The intensity factors (IFs) and energy release rate (ERR) are derived explicitly.
\end{abstract}

Keywords: Screw dislocation; piezoelectric bimaterials; edge-crack; interfacial fracture; intensity factors (IFs); energy release rate (ERRs); strips; conformal mapping; butt joint

\section{Introduction}

The study of dislocations in solid materials plays important role in fracture mechanics since dislocation solutions can serve as Green's functions to construct the solutions for various crack configurations under arbitrary loadings. In the last two decades, a number of investigations have been contributed to this field [1-4]. With extensive applications of piezoelectric ceramics in actuators, sensors, and transducers etc., the study of fracture and failure of piezoelectric materials has attracted numerous researchers' attention in recent years, especially the behaviors of various defects and inclusions under the coupled electric and mechanical loadings. For example, Deeg [5] first studied the response of piezoelectric solids with a dislocation, a crack, and an inclusion under coupled electromechanical fields, and Suo et al. [6] discussed the general solutions to collinear interfacial cracks in anisotropic piezoelectric bimaterials, etc. Detailed review of recent developments in fracture mechanics of piezoelectric materials can be found in the review paper by Zhang et al. [7]. In the study of screw dislocations in piezoelectric ceramics, Pak [8] first considered the Peach-Koehler forces acting on a screw dislocation under external electromechanical loadings. Lee et al. [9] and Chen et al. [10] dealt with a screw dislocation interacting with a semi-infinite crack in a transversely homogenous piezoelectric ceramics, and Kwon and Lee [11] further considered the similar case of a finite crack. Recently, by means of superposition, Soh [12,13], Wu et al. [14-16], and Liu et al. [17,18] obtained the fundamental solutions for screw dislocations in cracked piezoelectric bimaterial media with finite dimensions.

In reality, cracks more likely appear near free edges of bonded dissimilar blocks where stress 
concentration exists even without cracks. In this work, we consider a simple continuum model of screw dislocation interacting with an interfacial edge-crack in a piezoelectric bimaterial strip. By means of conformal mapping technique and known fundamental solutions to screw dislocations in piezoelectric bimaterials $[12,15]$, the out-of-plane displacement and in-plane electric potentials of the cracked strip are derived in closed-form. The intensity factors (IFs) and the energy release rate (ERR) of the edge-cracks are obtained explicitly.

\section{Problem statement and solution procedure}

As discussed in [8,9], the piezoelectric medium is supposed to be transversely isotropic, which has an isotropic basal plane parallel to $x y$ plane and a poling direction perpendicular to $x y$ plane. The boundary-value problem is treated as in the case of out-of-plane mechanical displacement and in-plane electric fields such that

$$
\begin{aligned}
& u_{x}=u_{y}=0, u_{z}=u_{z}(x, y), \\
& E_{x}=E_{x}(x, y), E_{y}=E_{y}(x, y), E_{z}=0 .
\end{aligned}
$$

In this case the constitutive relations reduce to

$$
\begin{aligned}
& \sigma_{x z}=c_{44} u_{z, x}+e_{15} \phi_{, x}, \quad D_{x}=e_{15} u_{z, x}-\varepsilon_{11} \phi_{, x}, \\
& \sigma_{y z}=c_{44} u_{z, y}+e_{15} \phi_{, y}, D_{y}=e_{15} u_{z, y}-\varepsilon_{11} \phi_{, y},
\end{aligned}
$$

where $\sigma_{\mathrm{kz}}, D_{\mathrm{k}}(k=x, y), c_{44}, e_{15}, \varepsilon_{11}$ and $\phi$ are the stress tensor, electric displacement vector, elastic modulus at constant electric field, piezoelectric constants, and electric potential, respectively. The electric field is given by

$E_{x}=-\phi_{, x}, E_{y}=-\phi_{, y}$

The governing equations are

$$
\sigma_{x z, x}+\sigma_{y z, y}=0, D_{x, x}+D_{y, y}=0
$$

Substitution of (3) into (5) leads to

$$
\begin{aligned}
& c_{44} \nabla^{2} u_{z}+e_{15} \nabla^{2} \phi=0, \\
& e_{15} \nabla^{2} u_{z}-\varepsilon_{11} \nabla^{2} \phi=0 .
\end{aligned}
$$

The above equations may be reduced as

$\nabla^{2} u_{z}=0, \nabla^{2} \phi=0$

As a result, the out-of-plane displacement and in-plane electric potentials can be expressed as the imaginary parts of two analytic functions $\mathrm{U}(\mathrm{z})$ and $\Phi(\mathrm{z})$, i.e.

$u_{z}=\operatorname{Im}[\mathrm{U}(z)], \phi=\operatorname{Im}[\Phi(z)]$,

where $\operatorname{Im}()$ denotes the imaginary part of an analytic function. It is convenient to introduce the complex stress and electric displacement:

$$
\begin{aligned}
& \sigma_{z y}+i \sigma_{z x}=c_{44}\left(u_{z, y}+i u_{z, x}\right)+e_{15}\left(\phi_{, y}+i \phi_{, x}\right), \\
& D_{y}+i D_{x}=e_{15}\left(u_{z, y}+i u_{z, x}\right)-\varepsilon_{11}\left(\phi_{, y}+i \phi_{, x}\right) .
\end{aligned}
$$

Using (8), relation (9) may be rewritten as

$$
\begin{aligned}
& \sigma_{z y}+i \sigma_{z x}=c_{44} \mathrm{U}^{\prime}(z)+e_{15} \Phi^{\prime}(z), \\
& D_{y}+i D_{x}=e_{15} \mathrm{U}^{\prime}(z)-\varepsilon_{11} \Phi^{\prime}(z) .
\end{aligned}
$$

Reserving the $x y$-coordinate system for the problem to be solved, we first determine the outof-plane displacement and in-plane electric potentials for a screw dislocation located in a cracked bimaterial, as shown in Fig. 1. The crack surfaces are assumed to be traction-free and electric impermeable. The screw dislocation is assumed located at $\zeta_{0}\left(\zeta_{0}=\xi_{0}+i \eta_{0}\right)$ in the lower half-plane and characterized by Burgers vector $b$, line-force $p$, line-charge $q$, and electric potential jump $\Delta \phi$. The complex potentials for this problem have been determined $[12,15]$ as

$$
\left[\begin{array}{c}
\mathrm{U}(\varsigma) \\
\Phi(\varsigma)
\end{array}\right]^{\prime}=\left\{\begin{array}{c}
2\left(L_{1}+L_{2}\right)^{-1} L_{2} T /\left(\varsigma-\varsigma_{0}\right)+2 L_{1}^{-1} h(\varsigma) \\
\varsigma \in D_{1} \text { i.e. }(\eta>0), \\
\left(L_{1}+L_{2}\right)^{-1}\left(L_{1}-L_{2}\right) \bar{T} /\left(\bar{\varsigma}-\bar{\varsigma}_{0}\right) \\
+T /\left(\varsigma-\varsigma_{0}\right)+2 L_{2}^{-1} h(\varsigma), \\
\varsigma \in D_{2} \text { i.e. }(\eta<0),
\end{array}\right.
$$


where

$$
\begin{aligned}
\mathbf{h}(\varsigma) & =B^{-1} / 2\left\{\mathbf{T} /\left(\varsigma-\varsigma_{0}\right)\left[1-\left(\varsigma_{0} / \varsigma\right)^{1 / 2}\right]\right. \\
& \left.+\overline{\mathbf{T}} /\left(\varsigma-\bar{\varsigma}_{0}\right) 1-\left(\bar{\varsigma}_{0} / \varsigma\right)^{1 / 2}\right\} .
\end{aligned}
$$

Here the prime denotes the derivative of an analytic function with respect to $\zeta=\xi+\mathrm{i} \eta, D_{1}$ and $D_{2}$ denote the half-planes above and below respectively, $L_{1}$ and $L_{2}$ represent the material matrices of the half-planes above and below, $B$ is the bimaterial matrix, and $\mathbf{T}$ is the complex quantity of the screw dislocation:

$$
\begin{aligned}
& L=\left[\begin{array}{cc}
c_{44} & e_{15} \\
e_{15} & -\varepsilon_{11}
\end{array}\right], B=\left(L_{1}^{-1}+L_{2}^{-1}\right), \\
& T=1 /(2 \pi)[b, \Delta \phi]^{T}+L_{2}^{-1} /(2 \pi i)[p,-q]^{T} .
\end{aligned}
$$

In the $x y$-coordinate system as shown in Figs. 2 and 3, the IFs are defined as [15]

$$
\mathbf{K}=\sqrt{2 \pi} \lim _{x \rightarrow 0} x^{1 / 2}[\mathbf{h}(x)+\overline{\mathbf{h}}(x)]
$$

where $\mathbf{K}=\left\{\mathrm{K}_{\mathrm{III}}, \mathrm{K}_{\mathrm{D}}\right\}^{\mathrm{T}}$, and $\mathrm{K}_{\mathrm{D}}$ is the electric intensity factor.

The traction and electric displacement at the interface a distance $r$ ahead of the crack tip and the displacement and electric potential jumps a distance $r$ behind of the crack tip are expressed as

$$
\mathbf{t}(r)=\mathbf{K} / \sqrt{2 \pi \mathrm{r}}, \mathbf{d}(r)=B \mathbf{K} / \sqrt{2 \pi \mathrm{r}},
$$

with

$\mathbf{t}=\left[\begin{array}{c}\sigma_{y z} \\ D_{y}\end{array}\right], \mathbf{d}=\left[\begin{array}{l}u_{z}\left(x, 0_{+}\right) \\ \phi\left(x, 0_{+}\right)\end{array}\right]-\left[\begin{array}{l}u_{z}\left(x, 0_{-}\right) \\ \phi\left(x, 0_{-}\right)\end{array}\right]$.

The ERR for a unit crack growth along the interface can be evaluated as

$$
\begin{aligned}
G & =\lim _{\Delta \rightarrow 0} 1 /(2 \Delta) \int_{0}^{\Delta} \mathbf{t}^{\mathrm{T}}(\Delta-r) \bullet \mathbf{d}(r) d r \\
& =(1 / 4) \mathbf{K}^{\mathrm{T}} B \mathbf{K} .
\end{aligned}
$$

Due to the out-of-plane displacement and inplane electric potentials satisfying two decoupled Laplace equations (7), conformal mapping technique is applicable for solving some interfacial edge-cracks in piezoelectric bimaterial strips.

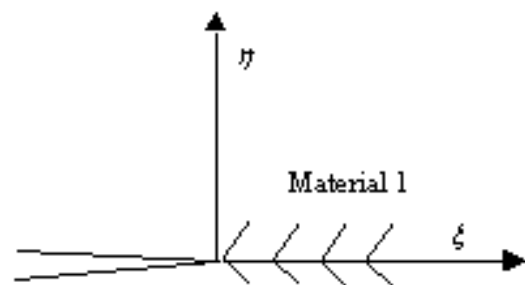

$\otimes$

Material 2

$\zeta_{l}=\xi_{1}+i_{t}$

$\xi=\xi+i \hbar$

Fig. I screw dislocation in cracked viezoelectric bimaterial

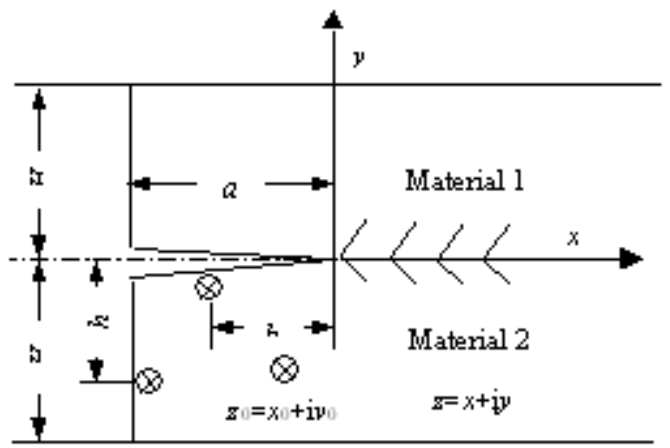

Fig. $2 \mathrm{~S}$ crew dis location in edge-cracked piezolectric strip

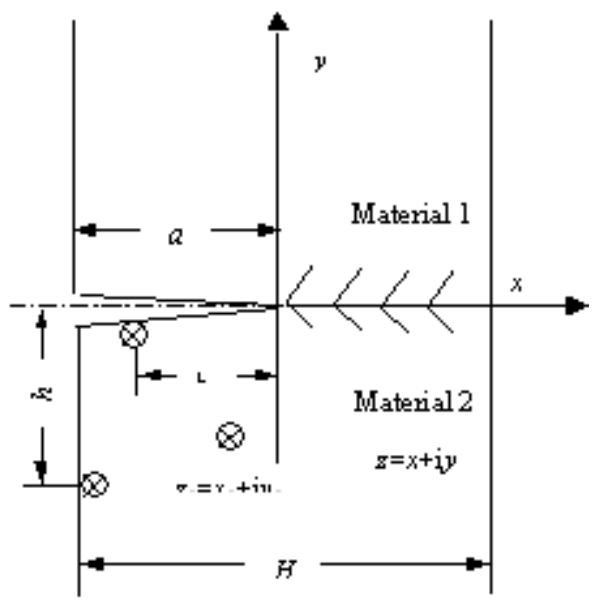

Fig. 3 Screw dislocation in edge-crack piezoelectric butt joint 


\section{Edge-cracked piezoelectric bimaterial strips}

Consider a screw dislocation interacting with an interfacial edge-crack between two sidebonded dissimilar piezoelectric strips of equal width, as shown in Fig. 2, in which $a$ and $H$ denote the crack length and the strip width, respectively. The crack surfaces and the edge surfaces are assumed to be traction-free and electric impermeable. The screw dislocation is located at $\mathrm{z}_{0}\left(\mathrm{z}_{0}=\mathrm{x}_{0}+\mathrm{iy}_{0}\right)$ in the lower strip.

Introduce the conformal mapping

$\zeta=f^{2}(z+a) / f^{2}(a)-1$

with

$$
f(z)=\sinh [\pi z /(2 H)] \text {, }
$$

which maps the bonded piezoelectric strips (Fig. 2) with an interfacial edge-crack onto two bonded half-planes with a semi-infinite cut along the negative $\xi$-axis, as shown in Fig. 1 .

Substitution of (18) and (19) into (11) and (12) leads to the potential of the strip. Its main contributor (12) may be expressed as

$$
\begin{aligned}
h_{1}(\mathrm{z})= & \pi B^{-1} /(4 H) \sinh [\pi(z+a) / H] \\
& \times\left\{T /\left[f^{2}(\mathrm{z}+a)-f^{2}\left(\mathrm{z}_{0}+a\right)\right]\right. \\
& \times\left[1-\left(\frac{f^{2}\left(z_{0}+a\right)-f^{2}(a)}{f^{2}(z+a)-f^{2}(a)}\right)^{1 / 2}\right] \\
& +\bar{T} /\left[f^{2}(\mathrm{z}+a)-\overline{f^{2}\left(\mathrm{z}_{0}+a\right)}\right] \\
& \left.\times\left[1-\left(\frac{f^{2}\left(z_{0}+a\right)}{f^{2}(z+a)-f^{2}(a)}\right)^{1 / 2}\right]\right\} .
\end{aligned}
$$

By the definition (14), the IFs are evaluated as

$$
\begin{aligned}
& \mathbf{K}=2 \sqrt{2} \pi B^{-1} / \sqrt{\pi a} \sqrt{\pi a / H \sinh (\pi a / H)} \\
& \times \operatorname{Re}\left\{-i \mathbf{T} / \sqrt{\cosh (\pi a / H)-\cosh \left[\pi\left(z_{0}+a\right) / H\right]}\right\},
\end{aligned}
$$

where $\mathbf{T}$ is the complex quantity of the screw dislocation defined in (13). By letting $H \rightarrow \infty$ in (21), we obtain the IFs for an interfacial edgecrack between two bonded dissimilar piezoelectric quarter-planes as

$$
\mathbf{K}=4 \pi B^{-1} / \sqrt{\pi a} \operatorname{Re}\left\{-i \mathbf{T a} / \sqrt{a^{2}-\left(a+z_{0}\right)^{2}}\right\} \text {. }
$$

Furthermore, by letting $a \rightarrow \infty$ in (21), we get the IFs for a semi-infinite crack between two infinite side-bonded piezoelectric strips as

$$
\mathbf{K}=2 \sqrt{2} \pi B^{-1} / \sqrt{H} \operatorname{Re}\left[-i \mathbf{T} / \sqrt{1-\exp \left(\pi z_{0} / H\right)}\right]
$$

As shown in Fig. 2, here we further consider two special loading cases of a line-force $P_{1}$ and a line-charge $Q_{1}$ located at $z_{0}=-b-0 i(0<\mathrm{b}<\mathrm{a})$ and a line-force $P_{2}$ and a line-charge $Q_{2}$ located at $z_{0}=$ $a$-i $h(h<H)$, respectively. Then, $\mathbf{T}$ may be rewritten as

$$
\mathbf{T}_{1}=L_{2}^{-1} /(2 \pi i)\left[P_{1},-Q_{1}\right]^{T}, \mathbf{T}_{2}=L_{2}^{-1} /(2 \pi i)\left[P_{2},-Q_{2}\right]^{T} .
$$

With the aid of (17) and (21), the IFs and ERR are evaluated respectively as

$$
\begin{aligned}
& \mathbf{K}=\sqrt{2} B^{-1} L_{2}^{-1} / \sqrt{\pi a} \sqrt{\pi a / H \sinh (\pi a / H)} \\
& \div \sqrt{\cosh [\pi a /(2 H)]-\cosh [\pi(a-b) / H]}\left[-P_{1}, Q_{1}\right]^{T},
\end{aligned}
$$

and

$$
\begin{aligned}
\mathrm{G} & =\frac{1}{2 H} \frac{\sinh (\pi a / H)}{\cosh [\pi a /(2 H)]-\cosh [\pi(a-b) /(2 H)]} \\
& \times\left[-P_{1}, Q_{1}\right] L_{2}^{-1} B^{-1} L_{2}^{-1}\left[-P_{1}, Q_{1}\right]^{T}
\end{aligned}
$$

for $\mathrm{z}_{0}=-b-0 i$, and

$$
\begin{aligned}
\mathbf{K} & =\sqrt{2} B^{-1} L_{2}^{-1} / \sqrt{\pi a} \sqrt{\frac{\pi a / H \sinh (\pi a / H)}{\cosh (\pi a / H)-\cos (\pi h / H)}} \\
& \times\left[-P_{2}, Q_{2}\right]^{T}
\end{aligned}
$$

and

$$
\begin{aligned}
\mathrm{G} & =\frac{1}{2 H} \frac{\sinh (\pi a / H)}{\cosh (\pi a / H)-\cos (\pi h / H)} \\
& \times\left[-P_{2}, Q_{2}\right] L_{2}^{-1} B^{-1} L_{2}^{-1}\left[-P_{2}, Q_{2}\right]^{T},
\end{aligned}
$$

for $\mathrm{z}_{0}=-a-\mathrm{i} h$.

If letting $H \rightarrow \infty$, results (25)-(28) cover those given by Li and Fan [19], who used the method of dual integral equations.

Furthermore, integration of (25) with respect to $b$ in the interval $[0$, a] leads to the solutions for a Griffith crack embedded at the mid-plane of a piezoelectric layer under out-of-plane mechanical and in-plane electric loading as 
those discussed by Li and Duan [20,21] and Li [22].

\section{Interfacial edge-crack in piezoelectric bimaterial butt joint}

Now let us consider the second case of a screw dislocation interacting with an interfacial edge-crack in a piezoelectric bimaterial butt joint, as shown in Fig. 3. Here $a$ and $H$ denote the crack length and the strip width, respectively. As aforementioned, the crack surfaces and the edge surfaces are assumed to be traction-free and electric impermeable. The screw dislocation is supposed located at $\mathrm{z}_{0}\left(\mathrm{z}_{0}=x_{0}+\mathrm{i} y_{0}\right)$ in the lower strip. In this case, we choose the conformal mapping

$$
\zeta=g^{2}(z+a) / g^{2}(a)-1
$$

with

$g(z)=\tan [\pi z /(2 H)]$,

which maps the cracked butt joint (Fig. 3) onto two bonded half-planes with a semi-infinite cut along the negative $\xi$-axis, as shown in Fig. 1 . Substituting (29) and (30) into (11) and (12), we obtain the potential for this problem. Its main contributor (12) may be expressed in terms of

$$
\begin{aligned}
& \mathbf{h}_{2}(\mathrm{z})=\pi B^{-1} /(2 H) \sec ^{2}[\pi(z+a) /(2 H)] \\
& \times\left\{g(z+a) \mathbf{T} /\left[g^{2}(z+a)-g^{2}\left(z_{0}+a\right)\right]\right. \\
& \times\left[1-\left(\frac{g^{2}\left(z_{0}+a\right)-g^{2}(a)}{g^{2}(z+a)-g^{2}(a)}\right)^{1 / 2}\right] \\
& +g(z+a) \overline{\mathbf{T}} /\left[g^{2}(\mathrm{z}+a)-\overline{g^{2}\left(\mathrm{z}_{0}+a\right)}\right] \\
& \left.\times\left[1-\left(\frac{\overline{g^{2}\left(z_{0}+a\right)}-g^{2}(a)}{g^{2}(z+a)-g^{2}(a)}\right)^{1 / 2}\right]\right\} \text {. }
\end{aligned}
$$

With the help of definition (14), we obtain the IFs as

$$
\begin{aligned}
& \mathbf{K}=4 \pi B^{-1} / \sqrt{\pi a} \sec [\pi a /(2 H)] \\
& \times \operatorname{Re}\{-i \mathbf{T} \sqrt{\pi a /(2 H) \tan [\pi a /(2 H)]} \\
& \left.\div \sqrt{\tan ^{2}[\pi a /(2 H)]-\tan ^{2}\left[\pi\left(z_{0}+a\right) /(2 H)\right]}\right\}
\end{aligned}
$$

By letting $H \rightarrow \infty$, (32) covers the IFs for an interfacial edge-crack between two bonded dissimilar piezoelectric quarter-planes as (22).

Furthermore, by letting $a \rightarrow \infty$ and simultaneously keeping $c=(H-a)$ constant, we have the IFs for a semi-infinite interfacial crack heading towards a free surface such that

$$
\mathbf{K}=4 \pi B^{-1} / \sqrt{\pi c} \operatorname{Re}\left[\mathbf{T}\left(c-\mathrm{z}_{0}\right) / \sqrt{z_{0}\left(2 c-z_{0}\right)}\right]
$$

Similar to Section 3, here we consider the same loadings shown in Fig. 3. With the aid of (24) and (32), the IFs and ERR are evaluated respectively as

$\mathbf{K}=2 / \sqrt{\pi a} \sec [\pi a /(2 H)]$

$$
\begin{aligned}
& \times \sqrt{\frac{\pi a /(2 H) \tan [\pi a /(2 H)]}{\tan ^{2}[\pi a /(2 H)]-\tan ^{2}[\pi(a-b) /(2 H)]}} \\
& \times B^{-1} L_{2}^{-1}\left[-P_{1}, Q_{1}\right]^{T}
\end{aligned}
$$

and

$$
\begin{aligned}
\mathrm{G}= & \frac{1}{2 H} \frac{\tan [\pi a /(2 H)] \sec ^{2}[\pi a /(2 H)]}{\tan ^{2}[\pi a /(2 H)]-\tan ^{2}[\pi(a-b) /(2 H)]} \\
& \times\left[-P_{1}, Q_{1}\right] L_{2}^{-1} B^{-1} L_{2}^{-1}\left[-P_{1}, Q_{1}\right]^{T}
\end{aligned}
$$

for $\mathrm{z}_{0}=-b-0 i$, and

$\mathbf{K}=2 / \sqrt{\pi a} \sec [\pi a /(2 H)]$

$\times \sqrt{\frac{\pi a /(2 H) \tan [\pi a /(2 H)]}{\tan ^{2}[\pi a / 2 H]+\tanh ^{2}[\pi h /(2 H)]}}$

$\times B^{-1} L_{2}^{-1}\left[-P_{2}, Q_{2}\right]^{T}$

and

$$
\begin{aligned}
\mathrm{G}= & \frac{1}{2 H} \frac{\tan [\pi a /(2 H)] \sec ^{2}[\pi a /(2 H)]}{\left.\tan ^{2}[\pi a /(2 H)]+\tanh ^{2}[\pi h /(2 H)]\right)} \\
& \times\left[-P_{2}, Q_{2}\right] L_{2}^{-1} B^{-1} L_{2}^{-1}\left[-P_{2}, Q_{2}\right]^{T}
\end{aligned}
$$

for $\mathrm{z}_{0}=-a-\mathrm{i} h$. By letting $H \rightarrow \infty$, results (34)-(37) again return to those given by $\mathrm{Li}$ and Fan [19] and Wu et al. [15].

Since the general potentials for the above crack configurations have been obtained, the entire electroelastic fields of the strips and the forces acting at the screw dislocation can be extracted explicitly based on the method discussed by Pak [8]. 


\section{Concluding remarks}

Explicit solutions to screw dislocation interacting with interfacial edge-crack between two bonded dissimilar piezoelectric strips have been determined in this work. Conformal mapping technique has been shown to be a powerful tool in solving some interfacial edgecracks in piezoelectric strips. Closed-form solutions (21) and (32) can be employed as a useful theoretical base for the assessment of numerical analyses, especially for estimating the effect of a/H ratio on the IFs and ERR of cracks in bonded piezoelectric structures. Furthermore, these explicit solutions can serve as Green's functions to construct the IFs and ERRs of piezoelectric strips with multiple cracks under arbitrary out-of-plane mechanical and in-plane electric loadings.

\section{Acknowledgement}

Partial support of this work by the U. S. Air Force Office of Scientific Research and the U. S. Army Research Office is gratefully acknowledged.

\section{References}

[1] Thomson, R. Physics of fracture, Solid State Physics, 39 (1986) 1-129

[2] Suo, Z. Singularities interacting with interface and cracks, International Journal of Solids and Structures, 25 (1989) 1133-1142

[3] Suo, Z. Singularities, interfaces and cracks in dissimilar anisotropic media, Proceedings of the Royal of Society of London, A427 (1990) 331-358

[4] Weertman, J. Dislocation Based Fracture Mechanics, World Scientific Publishing, Singapore, 1996

[5] Deeg, W.F. The Analysis of Dislocation, Crack, and Inclusion in Piezoelectric Solids, Ph.D. thesis, Stanford University, Stanford, CA, 1980

[6] Suo, Z., Kuo, C.-M., Barnett, D.M., Willis, J.R. Fracture mechanics for piezoelectric ceramics, Journal of the Mechanics and Physics of Solids, 40 (1992) 739-765

[7] Zhang, T.-Y, Zhao, M., Tong, P. Fracture of piezoelectric ceramics, Advances on Applied Mechanics, 38 (2001) 147-289

[8] Pak, Y.E. Force on a piezoelectric screw dislocation, ASME Journal of Applied Mechanics, 57 (1990) 863-869

[9] Lee, K.Y., Lee, W.G., Pak, Y. E. Interaction between a semi-infinite crack and a screw dislocation in a piezoelectric material, ASME Journal of Applied Mechanics, 67 (2000) 165-170
10] Chen, B.J, Xiao, Z.M., Liew, K.M., On the interaction between a semi-infinite anti-crack and a screw dislocation in piezoelectric solid, International Journal of Solids and Structures, 39 (2002) 1505-1513

[11] Kwon, K.K., Lee, K.Y. Electromechanical effects of a screw dislocation around a finite crack in a piezoelectric material, ASME Journal of Applied Mechanics, 69 (2002) 55-62

[12] Soh, A.K., Liu, J.X., Fang, D.N., A screw dislocation interacting with an interfacial crack in two dissimilar piezoelectric media, Physica Status Solidi B- Basic Research, 232 (2002) 273282

[13] Soh, A.K., Liu, J.X., Hoon, K.H., 3-D Green's functions for transversely isotropic magnetoelectroelastic solids, International Journal of Nonlinear Sciences and Numerical Simulation, 4 (2003) 139-148

[14] Wu, X.F., Dzenis Y. A., Zou W.S. Screw dislocation interacting with an interfacial edge crack between two bonded dissimilar piezoelectric wedges, International Journal of Fracture, 117 (2002) L9-L14.

[15] Wu, X.F., Cohn, S., Dzenis, Y.A. Screw dislocation interacting with interface and interfacial cracks in piezoelectric bimaterials, International Journal of Engineering Science, 41 (2003) 667-682

[16] Wu X.F., Dzenis, Y.A., Fan, T.Y. Screw dislocation interacting with twin interfacial edge cracks between two bonded dissimilar piezoelectric strips, Mechanics Research Communications, 30(2003) 547-555

[17] Liu, X.L., Liu, J.X., Liu, J. Green's function for a semi-infinite piezoelectric bimaterial strip with an interfacial edge crack. International Journal of Nonlinear Sciences and Numerical Simulation, 5 (2004) 61-66

[18] Liu, J.X., Screw dislocation in two dissimilar piezoelectric layers, Physica Status Solidi BBasic Research, 241 (2004) 298-304

[19] Li, X.F., Fan, T.Y. Mode-III interface edge crack between bonded quarter-planes of dissimilar piezoelectric materials, Archive of Applied Mechanics 71 (2001) 703-714

[20] Li, X.F, Duan, X.Y. Electroelastic analysis of a piezoelectric layer with electrodes, International Journal of Fracture, 111 (2001a) L73-L78

[21] Li, X.F, Duan, X.Y. Closed-form solution for a mode-III crack at the mid-plane of a piezoelectric layer, Mechanics Research Communications, 28 (2001b) 703-710

[22] Li, X.F. Electroelastic analysis of an anti-plane shear crack in a piezoelectric ceramic strip, International Journal of Solids and Structures, 39 (2002) 1097-1117 
